\title{
REPRESENTATIONS OF PRIME RINGS
}

\author{
BY \\ R. E. JOHNSON
}

This paper is a continuation of the study of prime rings started in [2]. We recall that a prime ring is a ring having its zero ideal as a prime ideal.

A right (left) ideal $I$ of a prime ring $R$ is called prime if $\mathfrak{a b} \subseteq I$ implies that $\mathfrak{a} \subseteq I$ (b $\subseteq I), \mathfrak{a}$ and $\mathfrak{b}$ right (left) ideals of $R$ with $\mathfrak{b} \neq 0(\mathfrak{a} \neq 0)$. We denote by $\mathfrak{P}_{\boldsymbol{r}}\left(\mathfrak{P}_{l}\right)$ the set of all prime right (left) ideals of $R$. For any subset $A$ of $R$, $A^{r}\left(A^{l}\right)$ denotes the right (left) annihilator of $A ; A^{r}\left(A^{l}\right)$ is a right (left) annihilator ideal of $R$. The set of all right (left) annihilator ideals of $R$ is denoted by $\mathfrak{A}_{r}\left(\mathfrak{A}_{l}\right)$.

For the prime rings $R$ studied in [2], it was assumed that there existed a mapping $I \rightarrow I^{*}$ of the set of all right (left) ideals of $R$ onto a subset $\Re(\Omega)$ of $\mathfrak{P}_{r}\left(\mathfrak{P}_{l}\right)$ having the following seven properties:

$$
\begin{aligned}
& I^{*} \supseteq I . \\
& \text { If } I \supseteq I^{\prime}, \text { then } I^{*} \supseteq I^{*} . \\
& \text { If } I \cap I^{\prime}=0, \text { then } I^{*} \cap I^{*}=0 . \\
& a I^{*} \subseteq(a I)^{*} \quad\left(I^{*} a \subseteq(I a)^{*}\right), \quad a \in R . \\
& \Re(\Omega) \text { is atomic. }
\end{aligned}
$$

$$
\begin{aligned}
& I^{* *}=I^{*} . \\
& 0^{*}=0 .
\end{aligned}
$$

That the above properties arise naturally may be seen by letting $I^{*}=p(I)$, the least prime right (left) ideal of $R$ containing $I$. Then properties (P1)-(P6) are known to hold [2]. Thus (P1)-(P7) hold for any ring having minimal prime right (left) ideals. In particular, these properties hold for a primitive ring with minimal right ideals.

A subset $\Re(\mathfrak{R})$ of $\mathfrak{B}_{r}\left(\mathfrak{P}_{l}\right)$ satisfying (P1)-(P7) will be called a right structure (left structure) of $R$. A right (left) structure $\Re(\mathfrak{R})$ of $R$ may be made into a lattice in the usual way. Thus for any $I, I^{\prime}$ in $\Re(\Omega)$, define $I \cap I^{\prime}$ as the intersection of these ideals and $I \cup I^{\prime}$ as $\left(I+I^{\prime}\right)^{*}$. It follows from [2] that $\Re(\mathfrak{R})$ is a modular lattice under these operations. A consequence of $[2, p .803]$ is that $\mathfrak{A}_{r} \subseteq \Re\left(\mathfrak{A}_{l} \subseteq \mathfrak{R}\right)$. Since $\left(I+I^{\prime}\right)^{l}=\left(I \cup I^{\prime}\right)^{l}$ by $(\mathrm{P} 6)$, it is evident that $\left(I \cup I^{\prime}\right)^{l}=I^{l} \cap I^{\prime l}$ for any $I, I^{\prime}$ in $\Re$, and similarly for $\&$.

It is assumed in this paper that the prime ring $R$ has both a right and a left structure. Some properties of structures, in addition to those given in [2], are developed in the first section. Next, atoms of these structures are used for dual representation spaces of $R$. It is shown that these structures in $R$ have isomorphic structures in their dual representation spaces. Finally, the

Presented to the Society, September 5, 1952; received by the editors May 24, 1952. 
given ring is shown to be an $n$-fold transitive ring of transformations on these spaces in a certain restricted sense.

1. Right-left structure relations. We assume that the prime ring $R$ has both a right structure $\Re$ and a left structure $\mathfrak{R}$. Each of the results of this section has a dual obtained by interchanging the roles of $\Re$ and $\mathbb{R}$.

1.1 Lemma. If $I$ is an atom of $\Re$ and $x$ is any nonzero element of $I$, then $(R x)^{*}$ is an atom of $\mathbb{R}$.

To prove this, let $L$ be any atom of $\mathfrak{R}$. The primeness of $R$ implies that $L \cap x R \neq 0$. Select $x a \in L \cap x R, x a \neq 0$; since $L$ is an atom, $(x a)^{l}$ is a maximal element of $\mathfrak{R}$ by $[2,4.11]$. Now $I \cap(x a)^{l r} \neq 0$, and therefore $I \subseteq(x a)^{l r}$. Thus $(x)^{l}=(x a)^{l}$ and $(R x)^{*}$ is an atom of $\mathfrak{R}$ by $[2,4.11]$.

The ring union of all atoms of $\Re$ is shown in $[2,4.2]$ to be an ideal of $R$. The above lemma shows that this ideal is also the ring union of all atoms of $\mathbb{R}$.

1.2 THEOREM. If $I$ is an atom of $\Re$, then $I^{l}$ is a maximal element of $\mathfrak{R}$, while if $I$ is a maximal element of $\Re$ for which $I^{l} \neq 0$, then $I^{l}$ is an atom of $\Omega$.

If $I$ is an atom of $\Re$, then $I^{l}=(x)^{l}$ for any nonzero $x$ in $I$, and hence $I^{l}$ is maximal in $\mathfrak{R}$ by the proof of the above lemma.

On the other hand, if $I$ is maximal in $\Re$ and $I^{l} \neq 0$, then $(x)^{r}=I$ for any nonzero $x$ in $I^{l}$. Thus $(x R)^{*}$ is an atom of $\Re$ by $[2,4.11]$. Since $x$ is in $(x R)^{*}$ $[2,1.2]$, we have by 1.1 that $(R x)^{*}$ is an atom of $\&$ for every nonzero $x$ in $I^{l}$. If $I^{l}$ is not an atom of $\mathfrak{R}$, it must contain atoms $L_{1}$ and $L_{2}$ such that $L_{1} \cap L_{2}$ $=0[2,4.3]$. Let $x_{1}$ be any nonzero element of $L_{1}$. Since $\left(x_{1}\right)^{l} L \neq 0$ due to the primeness of $R$, there must exist a nonzero element $x_{2}$ in $L_{2}$ such that $\left(x_{1}\right)^{2}$ $\neq\left(x_{2}\right)^{l}$. Then $R\left(x_{1}+x_{2}\right) \cap R x_{i} \neq 0, i=1,2$, and therefore $\left(R\left(x_{1}+x_{2}\right)\right)^{*}=\left(R x_{1}\right)^{*}$ $=\left(R x_{2}\right)^{*}$. This contradicts the assumption that $L_{1} \cap L_{2}=0$, and proves 1.2.

It is a corollary of 1.2 that the atoms of $\Re(\mathfrak{R})$ are contained in $\mathfrak{A}_{\boldsymbol{r}}\left(\mathfrak{A}_{l}\right)$.

1.3 THEOREM. If $I$ is an atom of $\Re$ and $I^{\prime}$ is any element of $\mathfrak{A}_{r}$, then $I \cup I^{\prime}$ also is in $\mathfrak{A}_{\text {r. }}$

Since $\left(I \cup I^{\prime}\right)^{l r}=\left(I^{l} \cap I^{\prime l}\right)^{r}$, what we wish to prove is that $\left(I^{l} \cap I^{\prime l}\right)^{r}$ $=I \cup I^{\prime}$. Clearly $I \cup I^{\prime} \subseteq\left(I^{\prime} \cap I^{\prime l}\right)^{r}$, so that we need only prove that $\left(I^{l} \cap I^{\prime l}\right)^{r}$ $\subseteq I \cup I^{\prime}$. In view of $[2,4.3]$, this can be accomplished by showing that every atom $I_{1}$ of $\Re$ contained in $\left(I^{l} \cap I^{\prime l}\right)^{r}$ is also contained in $I \cup I^{\prime}$.

So let us assume that $I_{1} \subseteq\left(I^{l} \cap I^{\prime l}\right)^{r}, I_{1}$ an atom of $\Re$. If either $I_{1}=I$ or $I_{1} \subseteq I^{\prime}$, nothing remains to be proved; henceforth we shall assume that $I_{1} \neq I$ and $I_{1} \Phi I^{\prime}$. Then necessarily $I \Phi I^{\prime}$ and $I^{\prime} \Phi I^{l}$. Hence there exists an atom $L$ of $\&[2,4.3]$ such that $L \subseteq I^{\prime l}, L \cap I^{l}=0$. Since $I^{l}$ is maximal in $\&$ by 1.2 , evidently $L \cup I^{l}=R$. From the modularity of $\&$ we see that $L \cup\left(I^{l} \cap I^{\prime l}\right)=I^{\prime l}$, and therefore that $L^{r} \cap\left(I^{l} \cap I^{\prime l}\right)^{r}=I^{\prime}$. Since $L^{r}$ is a maximal element of $\Re$ and $I \nsubseteq L^{r}$, clearly $I \cup L^{r}=R$. Hence it follows from (P5) that $I_{1} \cap\left(I+L^{r}\right) \neq 0$, and therefore that $\left(I_{1}+I\right) \cap L^{r} \neq 0$. Since also $I_{1}+I \subseteq\left(I^{l} \cap I^{\prime l}\right)^{r}$, it follows 
that $\left(I_{1}+I\right) \cap I^{\prime} \neq 0$. Thus $I_{1} \cap\left(I+I^{\prime}\right) \neq 0$ and $I_{1} \subseteq I \cup I^{\prime}$. This proves the theorem.

1.4 Corollary. If $I_{1}, \cdots, I_{n}$ are atoms of $\Re$, then $I_{1} \cup \cdots \cup I_{n}$ is in $\mathfrak{R}_{\text {r. }}$.

The corollary follows by mathematical induction.

2. $R$-modules. If $M$ is a right (left) $R$-module and $A$ is a subset of $M$, we shall again use the notation $A^{r}\left(A^{l}\right)$ to denote the annihilator of $A$ in $R$. A right (left) $R$-module $M$ is called prime if $A^{r}=0\left(A^{l}=0\right)$ for every nonzero submodule $A$ of $M$. A submodule $M^{\prime}$ of $M$ is called a prime submodule of $M$ if $M-M^{\prime}$ is a prime module. If the ring $R$ has a right (left) structure $\Re(R)$, then a right (left) $R$-module $M$ is called admissible relative to $\Re(R)$ if $M$ is prime and $(x)^{r} \in \Re\left((x)^{l} \in \mathfrak{R}\right)$ for every $x \in M$. For any $I$ in $\Re(\mathfrak{R})$, both $I$ and $R-I$ are examples of admissible right (left) $R$-modules.

It is shown in [2, p. 804] that an admissible right $R$-module $M$ has a structure much the same as $R$ does. For any submodule $N$ of $M$, define

$$
N^{*}=\left\{x ; x \in M,[(N: x)]^{*}=R\right\} .
$$

Here $(N: x)$ denotes the annihilator in $R$ of the element $x+N$ in $M-N$. Then the set $\mathfrak{M}$ of all submodules $N^{*}$ of $M$ is a structure of $M$ in that it possesses the properties analogous to (P1)-(P7). Naturally, similar remarks hold for admissible left $R$-modules.

Let us assume now that $R$ is a ring with a right structure $\Re$ and a left structure $\mathfrak{R}$, and that $N$ is a fixed atom of $\Re$. Select an atom $M$ of $\mathfrak{R}$ so that

$$
M \cdot N \neq 0 .
$$

Such an $M$ must exist, since the ring union $S$ of all atoms of $\mathfrak{R}$ is an ideal of $R[2,4.2]$, and $S \cdot N \neq 0$ due to the primeness of $R$. Let

$$
K=M \cap N,
$$

a nonzero subring of $R$. If we consider the rings $K, M$, and $N$ as modules, it is evident that $K$ is an $(N, M)$-module, that $M$ is an $(R, K)$-module, and that $N$ is an $(K, R)$-module. Clearly $N \cdot M \subseteq K$.

2.1 Lemma. For $x$ in $M$ and $y$ in $N, x y=0$ if and only if $x=0$ or $y=0$.

If $x \neq 0$, then $(x)^{r}$ is an element of $\Re$ and therefore either $(x)^{r} \cap N=0$, in which case the desired conclusion follows immediately, or $N \subseteq(x)^{r}$. In this latter case evidently $N^{l} \cap M \neq 0$ and $M \subseteq N^{l}$, which is contrary to the choice of $M$. This proves 2.1 .

An obvious corollary of this lemma is that $K$ is an integral domain.

2.2 Lemma. The integral domain $K$ possesses a quotient division ring $D$.

If $x$ and $y$ are nonzero elements of $K$, then $(x N)^{*}=(y N)^{*}=N$ in view of 2.1 and the fact that $N$ is an atom. Thus $x N \cap y N \neq 0$ by (P5), and 
hence $x N \cap y N \cap M \neq 0$. However, $x N \cap y N \cap M \subseteq K$ so that evidently $x K$ $\cap y K \neq 0$. This proves that $K$ has a right quotient division ring $D$. That $D$ also is the left quotient of $K$ follows by duality.

2.3 Lemma. If $x$ and $y$ are nonzero elements of $N$, then $K x \cap K y \neq 0$ if and only if $(x)^{r}=(y)^{r}$.

If $K x \cap K y \neq 0$, then obviously $(x)^{r}=(y)^{r}$. Conversely, if $(x)^{r}=(y)^{r}$, then $x$ and $y$ are in $(x)^{r l}$, an atom of $\mathbb{R}$. Now $M x \neq 0$ and $M y \neq 0$, and since both $M x$ and $M y$ are contained in the atom $(x)^{r l}$, necessarily $M x \cap M y \neq 0$ by (P5). Since $K$ is a right $M$-module, evidently $K x \cap K y \neq 0$ as desired.

We shall consider $K$ as having the trivial left and right structures, namely the structures consisting of the set $(0, K)$. In view of 2.2 , which guarantees that (P5) holds, it is evident that these structures satisfy (P1)-(P7).

Now $N$, as an admissible $(K, R)$-module, has a left structure induced by $K$ and a right structure induced by $R$. It is clear that for any $K$-submodule $A$ of $N$, the closure $A^{*}$ of $A$ is defined as follows:

$$
A^{*}=\{x ; x \in N, x=0 \text { or } K x \cap A \neq 0\} .
$$

If $\mathfrak{R}$ denotes the set of all closed $K$-submodules of $N$, then $\mathfrak{R}$ is a left structure of $N$. Since $N$ is an atom of $\Re$, the right structure of $N$ induced by $R$ is the trivial one.

In an analogous way, of course, $M$ has left and right structures induced by $R$ and $K$ respectively. The left structure is trivial; the right structure of $M$ induced by $K$ will be denoted by $\mathfrak{M}$.

The following results, although frequently just stated for $\mathfrak{R}$, have the obvious duals relative to $\mathfrak{M}$.

Any $A$ of $\mathfrak{R}$ is actually a left $N$-module. For if $x \in N$ and $a \in A$ with $x a \neq 0$, then $(x a)^{r}=(a)^{r}$ since both of these right ideals are maximal elements of $\Re$ by [2, 4.11], and $K(x a) \cap K a \neq 0$ by 2.3. Thus $K(x a) \cap A \neq 0$ and $x a \in A$ since $A^{*}=A$.

If $L$ is in $\ell$ and $k x$ is a nonzero element of $L \cap N, k \in K$ and $x \in N$, then $(R x)^{*} \cap L \neq 0$ and, since $(R x)^{*}$ is an atom of $\mathfrak{R}$ by 1.1 , evidently $(R x)^{*} \subseteq L$. Thus $x$ is in $L \cap N$ and we have proved that $L \cap N$ is in $\Re$. Furthermore, if $L$ is an atom of $\mathbb{R}$, then $L \cap N$ is an atom of $\mathfrak{R}$. This is so since for any nonzero elements $x$ and $y$ of $L \cap N,(x)^{r}=(y)^{r}=L^{r}$, and hence $K x \cap K y \neq 0$ by 2.3.

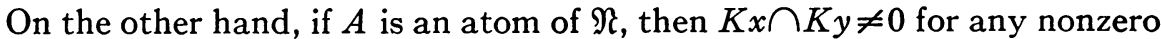
$x, y \in A$. Hence $A^{r l}=(x)^{r l}=L$, an atom of $\&$, and $A=L \cap N$.

The above remarks constitute part of the proof of the following theorem.

2.4 Theorem. The $K$-submodule $A$ of $N$ is in $\mathfrak{R}$ if and only if $A=L \cap N$ for some $L$ in $\&$.

To complete the proof of this theorem, let $A$ be any nonzero element of $\mathfrak{R}$ and let $L=(R A)^{*} \in \mathbb{R}$. We shall prove that $A=L \cap N$. If $L_{1} \subseteq L, L_{1}$ an atom of 
$\mathcal{Q}$, then $L_{1} \cap R A \neq 0$ so that $N \cdot\left(L_{1} \cap R A\right) \neq 0$ and $L_{1} \cap N R A \neq 0$. Since $N R A$ $\subseteq N A \subseteq A$, we have proved that $L_{1} \cap A \neq 0$. Now $L_{1} \cap N$ is an atom of $\mathfrak{R}$ and therefore $L_{1} \cap N \subseteq A$. It follows that $L \cap N \subseteq A$, and the proof of the theorem is completed.

2.5 Theorem. The lattices $\{\Omega ; \subseteq, \cup, \cap\}$ and $\{\Re ; \subseteq, \cup, \cap\}$ are isomorphic under the correspondence $L \rightarrow L \cap N$. Dually, the lattices $\{\Re ; \subseteq, \cup, \cap\}$ and $\{\mathfrak{M} ; \subseteq, \cup, \cap\}$ are isomorphic under the correspondence $I \rightarrow I \cap M$.

It is sufficient to prove that the mapping $L \rightarrow L \cap N$ of $\mathfrak{R}$ onto $\mathfrak{R}$ is a 1-1 order-preserving mapping in order to prove that these lattices are isomorphic. Clearly the mapping is order-preserving. In order to show that it is a 1-1 mapping, we need only note that if $L_{1} \nsubseteq L_{2}, L_{i} \in \mathcal{R}$, then there exists an atom $L$ of $\&$ such that $L \subseteq L_{1}, L \cap L_{2}=0$ by $[2,4.3]$. Hence $L \cap N \Phi L_{2} \cap N$ and therefore $L_{1} \cap N \Phi L_{2} \cap N$. This proves 2.5 .

In case $R$ is a primitive ring with nonzero socle $S$, and $N$ and $M$ are simple conjugate right and left $R$-modules respectively with common centralizer $D$, this theorem yields the well known isomorphism existing between the lattice of left (right) ideals of $S$ and the lattice of $D$-submodules of $N(M)$. This application to primitive rings is obtained by letting $\Re(\mathfrak{R})$ be the set of all prime right (left) ideals of $R$.

If $A \in \mathfrak{N}$, say $A=L \cap N$ for $L \in \mathcal{R}$, then obviously $A^{r} \supseteq L^{r}$. Since, however, $A^{r l} \in \mathcal{R}$ and $A^{r l} \supseteq A$, evidently $L \subseteq A^{r l}$ and $A^{r} \subseteq L^{r}$. Thus $A^{r}=L^{r}$. If, in particular, $L \in \mathfrak{A}_{l}$, then $A=A^{r l} \cap N$. Let us denote by $\mathfrak{N}_{l}\left(\mathfrak{M}_{r}\right)$ the set of all $K$-submodules of $N(M)$ that are annihilators of right (left) ideals of $R$. Then $\mathfrak{N}_{l}=\left\{A ; A \in \mathfrak{N}, A=L \cap N\right.$ for some $\left.L \in \mathfrak{A}_{l}\right\}$, and similarly for $\mathfrak{M}_{r}$.

In view of the isomorphism existing between $\mathfrak{R}$ and $\mathfrak{R}$, Theorem 1.3 has the following counterpart in $\mathfrak{R}$.

2.6 Theorem. If $A$ is an atom of $\mathfrak{N}$ and $B$ is any element of $\mathfrak{R}_{l}$, then $A \cup B$ also is in $\mathfrak{N}_{l}$.

A corollary of this theorem is as follows (1.4):

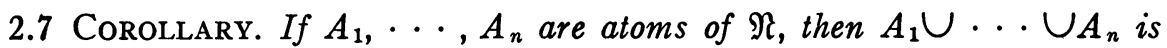
in $\mathfrak{N}_{l}$.

For a primitive ring $R$, analogues of Theorem 2.6 and its corollary can be found in a recent paper by Artin [1, pp. 68, 69]. His results are more general than ours in that his ring $R$ is not assumed to have minimal right ideals. Of course, they are also less general in that they are restricted to apply to primitive rather than prime rings.

3. Transitivity of $R$ over $N$. As usual, the elements $x_{1}, \cdots, x_{n}$ of $N$ are called $K$-linearly independent if and only if $k_{1} x_{1}+\cdots+k_{n} x_{n}=0$ implies all $k_{i}=0, k_{i} \in K$. An alternate lattice-theoretic definition is given by the following lemma. 
3.1 LEMмA. The elements $x_{1}, \cdots, x_{n}$ of $N$ are $K$-linearly independent if and only if

$$
\left(x_{j}\right)^{r} \nsupseteq \bigcap_{i=1, i \neq j}^{n}\left(x_{i}\right)^{r}, \quad j=1, \cdots, n .
$$

To prove this lemma, note first that all $A_{i}=\left(x_{i}\right)^{r l} \cap N=\left(K x_{i}\right)^{*}$ are atoms of $\mathfrak{N}$ (assuming, of course, that $x_{i} \neq 0$ ). If $k_{1} x_{1}+\cdots+k_{n} x_{n}=0$ with $k_{j} \neq 0$, then

$$
A_{j} \subseteq \bigcup_{i=1, i \neq j}^{n} A_{i}
$$

and, since $A_{i}^{r}=\left(x_{i}\right)^{r}$,

$$
\left(x_{j}\right)^{r} \supseteq \bigcap_{i=1, i \neq j}^{n}\left(x_{i}\right)^{r}
$$

Conversely, if the above inclusion relation holds for some $j$, then

$$
\begin{gathered}
A_{j} \subseteq \bigcup_{i=1, i \neq j}^{n} A_{i}, \\
K x_{j} \cap \sum_{i=1, i \neq j}^{n} K x_{i} \neq 0 .
\end{gathered}
$$

Thus the elements $x_{1}, \cdots, x_{n}$ are $K$-linearly dependent, and 3.1 follows.

3.2 LEMмA. Let $I$ be any right ideal of $R$ and $K^{\prime}$ be any left $N$-submodule of $K$. Then for any $x$ and $y$ in $N$ such that $x I \neq 0$ and $K^{\prime} y \neq 0$, also $x I \cap K^{\prime} y \neq 0$.

To prove this lemma, let $k$ be any nonzero element of $K^{\prime}$. Then, by the primeness of $N, x a k y \neq 0$ for some $a$ in $I$. Now $x(a k y)=(x a k) y$ where $a k y \in I$ and $x a k \in K^{\prime}$, and therefore the lemma is proved.

We now are in a position to prove the main result of our paper, namely that $R$ acts almost as an $n$-fold transitive ring of $K$-linear transformations on $N$ for any integer $n$ not exceeding the $K$-dimension of $N$. To be more precise, we shall prove the following theorem.

3.3 Transitivity Theorem. If $x_{1}, \cdots, x_{n}$ is any set of $n K$-linearly independent elements of $N$ and if $y_{1}, \cdots, y_{n}$ is any set of $n$ elements of $N$, then there exist an element $a$ of $R$ and a nonzero element $k$ of $K$ such that

$$
x_{i} a=k y_{i},
$$$$
i=1, \cdots, n \text {. }
$$

To aid in the proof of this theorem, let

$$
I_{j}=\bigcap_{i=1, i \neq j}^{n}\left(x_{i}\right)^{r}
$$


In view of 3.1, evidently $I_{j} \Phi\left(x_{j}\right)^{r}$ for any $j$. Hence, by 3.2, there exist elements $a_{j} \in I_{j}$ and $k_{j} \in K, k_{j} \neq 0$, such that $x_{j} a_{j}=k_{j} y_{j} \neq 0$ for all $j$ such that $y_{j} \neq 0$. If $y_{j}=0$, select $a_{j}=0$. Now for all $y_{j} \neq 0, k_{j} y_{j} K$ is a right ideal of $K$, and $\bigcap_{j} k_{j} y_{j} K \neq 0$ by 2.2 . Select $k \in \bigcap_{j} k_{j} y_{j} K, k \neq 0 ; k=k_{j} y_{j} c_{j}$ for each $j$ such that $y_{j} \neq 0$. Then $x_{j}\left(a_{j} c_{j} y_{j}\right)=k y_{j}$, and if we let $a=a_{1} c_{1} y_{1}+\cdots+a_{n} c_{n} y_{n}$, evidently $x_{j} a=k y_{j}$ as desired.

We give now an example of a prime ring of the type considered in this paper. Denote by $I$ the ring of integers and by $I_{2}$ the ring of all $2 \times 2$ matrices over $I$. We use the notation $E_{i j}$ for the matrix with 1 in its $(i, j)$ position and zeros elsewhere. Now denote by $R$ the set of all matrices of $I_{2}$ having all even or all odd integers for components. It is easily established that $R$ is a prime ring.

The right ideal $N=2 I E_{11}+2 I E_{12}$ is a minimal prime right ideal and the left ideal $M=2 I E_{11}+2 I E_{21}$ is a minimal prime left ideal. Clearly $K=M \cap N$ $=2 I E_{11}$ is an integral domain. The sets of prime right and left ideals of $R$ form right and left structures of $R$.

As an illustration of the transitivity theorem, let $x_{1}=2 E_{11}, x_{2}=4 E_{12}$, $y_{1}=0$, and $y_{2}=2 E_{11}$. Then for $a=2 E_{11}$ and $k=4 E_{11}$ we have $x_{1} a=k y_{1}$ and $x_{2} a=k y_{2}$. We note that there is no $a$ in $R$ such that $x_{1} a=y_{1}$ and $x_{2} a=y_{2}$.

In the case of a primitive ring $R$, the minimal right ideals are all isomorphic as right $R$-modules. That such is not the case in general for a prime ring follows from this example. To show this, let $N^{\prime}=\left[I\left(E_{11}+E_{21}\right)\right.$ $\left.+I\left(E_{12}+E_{22}\right)\right] \cap R$. It is not too difficult to show that $N^{\prime}$ is a minimal prime right ideal of $R$. If $N$ and $N^{\prime}$ were isomorphic, then we would have $2 a E_{11}$ $\rightarrow c\left(E_{11}+E_{21}\right), 2 b E_{12} \rightarrow d\left(E_{12}+E_{22}\right)$ for some integers $a, b, c, d$ in order for the annihilators of corresponding elements of $N$ and $N^{\prime}$ to be the same. But then $c$ and $d$ would have to be even integers, and nothing in $N$ would correspond to the matrices in $N^{\prime}$ having odd integers for elements.

\section{BIBLIOGRAPHY}

1. E. Artin, The influence of J.H. M. Wedderburn on the development of modern algebra, Bull. Amer. Math. Soc. vol. 56 (1950) pp. 65-72.

2. R. E. Johnson, Prime rings, Duke Math. J. vol. 18 (1951) pp. 799-809.

Smith College, NORTHAMPTON MASS. 